\title{
Initial Design and Optimization of Broad-Band and Dual-Band Square-to-Circular Waveguide Transitions
}

\author{
Jens Bornemann and Marjan Mokhtaari \\ Department of Electrical and Computer Engineering \\ University of Victoria, Victoria, BC, V8W 3P6, Canada
}

\begin{abstract}
This paper presents guidelines for the design of square-tocircular waveguide transitions. Based on an algorithm developed for rectangular waveguide transformers, performances of initial and optimized transitions are presented. Although a direct transition from square to circular waveguide provides already a reasonable return loss, it is shown that more sections are required for broad-band and dual-band transitions. Verification of design and analysis procedures is provided through comparison with the commercially available field solver HFSS.
\end{abstract}

Key Words - Waveguide transitions, waveguide discontinuities, satellite communications

\section{INTRODUCTION}

Transitions from square to circular waveguides are frequently required in front ends for dual-polarized satellite applications, e.g. [1] - [3]. While the design of rectangular-to-rectangular or circular-to-circular impedance transformers are well documented, e.g. [3], [4], transitions from rectangular to circular waveguides require special attention caused by different field configurations in the two guides, e.g. [5], [6]. Simple design guidelines for rectangular-to-circular transitions are presented in [7]. However, these expressions specifically require a rectangular waveguide section, which cannot be used in square-to-circular transitions operating in dual-polarization mode.

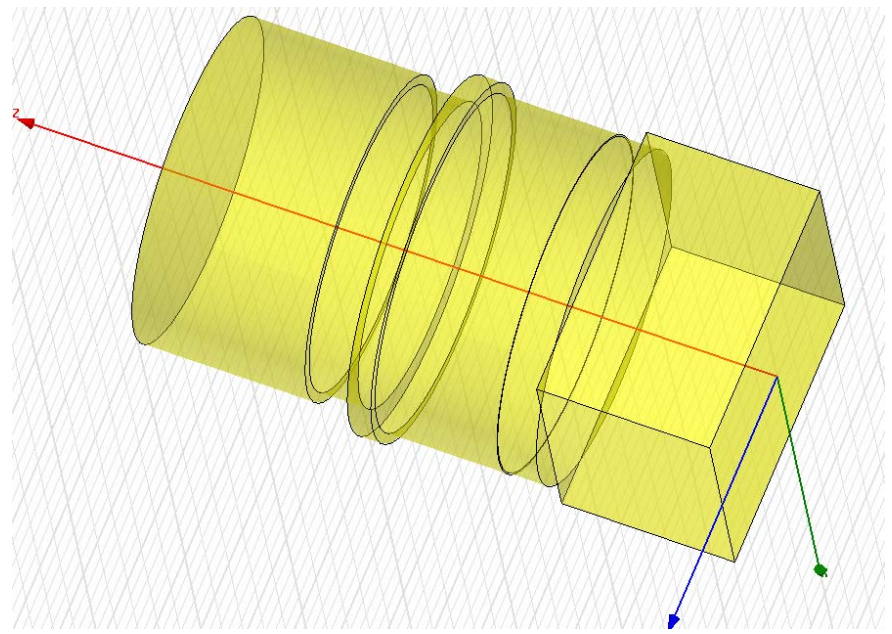

Figure 1. Principle layout of a square-to-circular waveguide transition.

Therefore, this paper presents initial design guidelines for square-to-circular waveguide transitions (e.g. Fig. 1) and demonstrates optimization results for broad-band and dual- band applications. The calculation of the initial dimensions is performed by a slight modification of a rectangular-torectangular impedance transformation procedure. Its advantages are twofold: First, it provides dimensions which immediately lead to return-loss values between 20 and $30 \mathrm{~dB}$; secondly, it provides enough sections which allow optimization towards more demanding specifications. The performance evaluation and optimization are achieved by using a code based on mode-matching techniques.

\section{DESIGN}

\section{A. Analysis}

For the field-theory-based analysis, the square waveguide is treated as a special case of the rectangular waveguide. Since the optimization procedure will drastically vary the widths/heights of square and the diameters of circular waveguides, a variety of different discontinuities between rectangular and circular waveguides must be considered. Within the framework of this paper, modal algorithms presented in [8] - [10] are used together with basic modal scattering matrix combinations of [3].

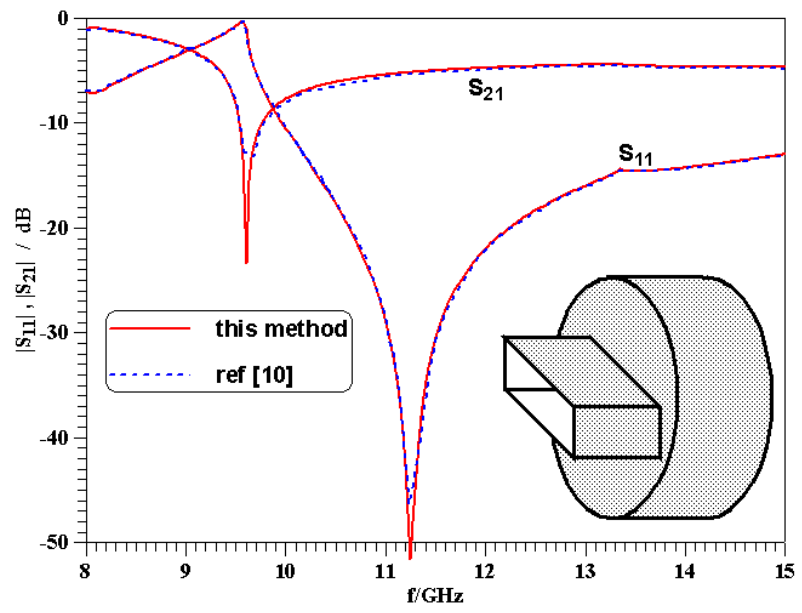

Figure 2. Performance comparison for a rectangular-to-circular discontinuity [10]

In order to verify the analysis code, we compare our results with those of [10] in Fig. 2. Note that higher-order mode 
propagation in the circular waveguide occurs above $9.6 \mathrm{GHz}$. Fig. 3 shows a comparison with an HFSS analysis of a structure originally proposed in [7]. Excellent agreement is observed in both cases, thus validating the analysis of rectangular/square-to-circular transitions.

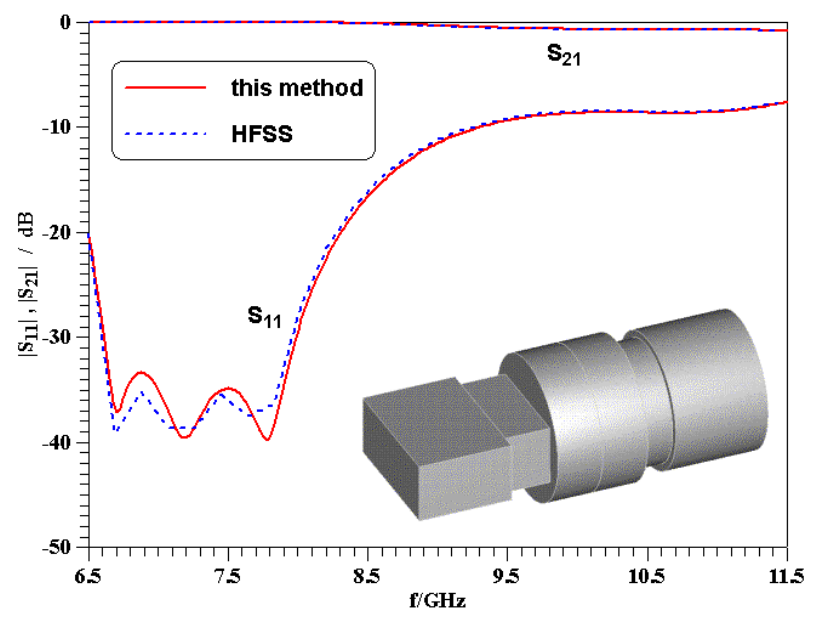

Figure 3. Performance comparison for a three-section rectangular-to-circular transition [7].

\section{B. Initial Design}

The initial design is based on the rectangular-to-rectangular transformer design in [3]. It starts with a linear stepping for the widths $a(i)$ and then uses a nonlinear relationship for the heights $b(i)$ to achieve impedance matching. The lengths $L(i)$ of the sections are adjusted to compensate for the individual discontinuity effects, e.g., [3], [4]. Due to this procedure, a square-to-square transformer will have a square transformer section only if a single transformer section is used. For a number of sections $N>1$, individual transformer sections will be rectangular rather than square.

In adapting this design procedure to square-to-circular transitions, we first assume that all transformer sections be circular as shown in the inset of Fig. 4. The diameters of the circular sections can now be related to $a(i)$ or $b(i)$ in the following ways.

First, the diameters can equal the widths of the rectangular design, i.e., $d(i)=a(i)$. However, since the widths are stepped linearly, this would not result in a different performance for more sections, as the differences in diameters would be marginal. This can be seen in Fig. 4. The curve for the direct connection $(N=0)$ is within the plotting accuracy of that with a single transformer section $(N=1)$. Any more sections will only increase the length but not result in a different return-loss behavior. It should be pointed out, however, that the direct connection between the square $(15.8 \mathrm{~mm} \times 15.8 \mathrm{~mm})$ and the circular $(18.5 \mathrm{~mm}$ diameter) waveguide results in a broadband performance, which will satisfy specifications for many applications, e.g. [2]. For the design examples shown here, we require a return loss of $28 \mathrm{~dB}$ between $11.7 \mathrm{GHz}$ and $18.1 \mathrm{GHz}$ as shown by the rectangle in Fig.4.
Secondly, the diameters of the circular sections can equal the heights of the rectangular design, i.e., $d(i)=\mathrm{b}(i)$. This turns out to yield rather large diameters, which cause resonance peaks to appear within the frequency band of operation (not shown here). Thirdly, a combination of $a(i)$ and $b(i)$ can be used to determine the diameters. In order to limit the influence of the rather large $b$ dimensions, we choose the geometric mean.

$$
d(i)=\sqrt{a(i) b(i)}
$$

The performances of initial designs obtained from this relationship are shown in Fig. 4 for $N=2$ and $N=4$. Although they only partly satisfy the specifications, it is obvious that there exist a potential for improvement through optimization.

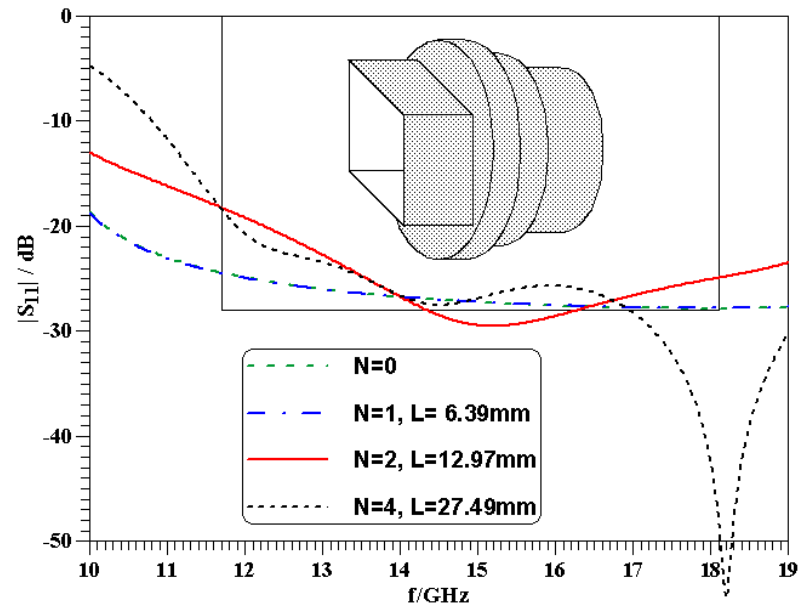

Figure 4. Performances of initial designs with $N=0$ (direct connection), $N=1, N=2$ (see inset) and $N=4$ transformer sections.

Design specifications $(-28 \mathrm{~dB})$ for this example are denoted by the rectangle. Note that graphs for $N=0$ and $N=1$ fall within the plotting accuracy (cf. text).

\section{Optimization}

Once an initial design has been obtained, the diameters and lengths of the circular sections between the square input and the circular output guide are optimized using a MiniMaxbased algorithm, e.g. [11]. The function to be minimized is

$$
F=\sum_{n=1}^{N_{f}} D_{r l}\left(f_{n}\right) / A_{r l}\left(f_{n}\right)
$$

where $D_{r l}\left(f_{n}\right)$ and $A_{r l}\left(f_{n}\right)$ are the desired and actual return loss, respectively, at frequency $f_{n}$. As an additional constraint for the optimization, we require the final design to be shorter than the initial one.

\section{RESULTS}

Fig. 5 shows the performance of optimized one- and twosection transitions for broad-band applications. Although a single section appears attractive from a fabrication point of view, there are not enough optimization parameters to satisfy the $28 \mathrm{~dB}$ return-loss specification. Very good agreement with HFSS is obtained for the two-section design. Note that the 
total length is not much longer than the one-section design, but shorter than the initial value shown in Fig. 4.

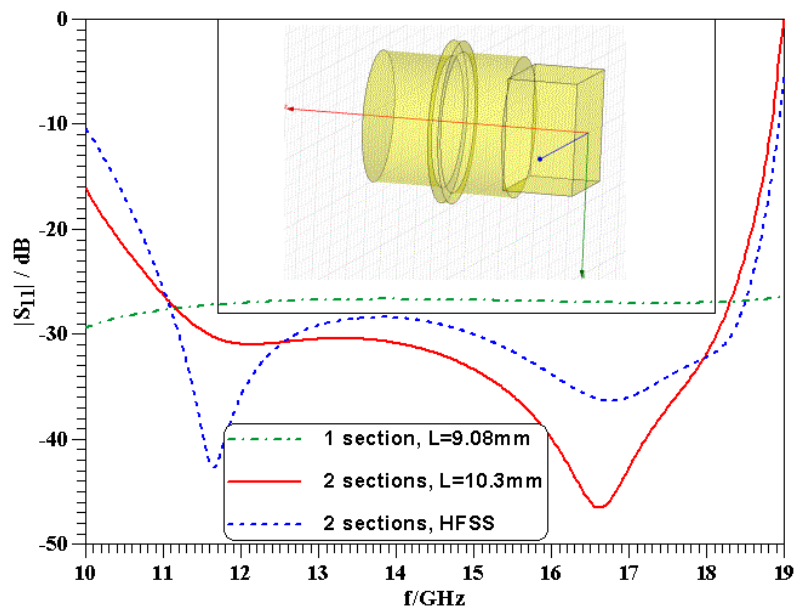

Figure 5. Broad-band one- and two-section square-to-circular transitions and comparison with HFSS.

A dual-band transition for $\mathrm{Ku}$-band satellite applications $(17.1-12.5 \mathrm{GHz}$ and $17.3-18.1 \mathrm{GHz},[12])$ is shown in Fig. 6. The $36 \mathrm{~dB}$ return-loss specification in both bands is met by an optimized four-section transition that is reduced in length by more than 50 percent compared to the initial design in Fig. 4. Very good agreement with HFSS is observed, thus validating the design procedure.

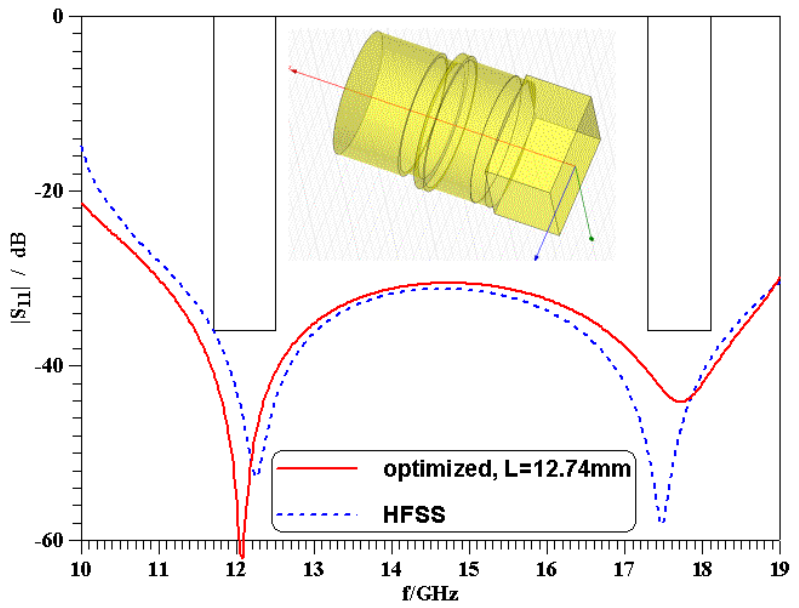

Figure 6. Dual-band four-section square-to-circular transition and comparison with HFSS.

\section{CONCLUSIONS}

The design guidelines presented in this paper offer an attractive solution for high-performance broad-band and dualband square-to-circular waveguide transitions. By using a profile for rectangular waveguide transformers and adapting the related dimensions to circular waveguides, initial dimensions are readily obtained and fine-optimized to fit stringent specifications, e.g., for dual-polarized satellite applications. Both the field-theoretical analysis and the design procedure are verified by comparisons with results from the literature and those obtained from the commercially available field solver HFSS.

\section{REFERENCES}

[1] T. Kitsuregawa, Advanced Technology in Satellite Communication Antennas - Electrical and Mechenical Design,. Artech House Inc., Norwood, 1990.

[2] J. M. Rebollar, J. de Frutos, "Dual-band compact square waveguide corrugated polarizer", in 1999 IEEE AP-S Int. Symp. Dig. pp. 962 - 965, Orlando, USA, July 1999.

[3] J. Uher, J. Bornemann and U. Rosenberg, Waveguide Components for Antenna Feed Systems - Theory and CAD. Artech House Inc., Norwood, 1993.

[4] G. L. Matthaei, L. Young and E. M. T. Jones, Microwave Filters, Impedance Matching Networks and Coupling Structures, McGraw-Hill, New York 1964.

[5] J. Huang, R. Vahldieck and H. Jin, "Frequency- domain TLM analysis of the transition from rectangular to circular waveguide", in 1994 IEEE MTT-S Int. Microwave Symp. Dig., pp. 705-708, San Diego, USA, May 1994

[6] M. Mongiardo and C. Tomassoni, "Modal analysis of discontinuities between elliptical waveguides", IEEE Trans. Microwave Theory Tech., vol. 48, pp. 597-605, Apr. 2000.

[7] U. Rosenberg, J. Bornemann and K. Rambabu, "Analysis and design of flange-integrated rectangular-to-circular waveguide transformers for splashplate antenna feeds", in Proc. $32^{\text {nd }}$ European Microwave Conf., pp. 601-604, Milan, Italy, Sep. 2002.

[8] H.D. Knetsch, "Contribution to the theory of abrupt cross-sectional changes in waveguides" (in German), Int. J. Electron. Commun. (AE ̈̈), vol. 22, pp. 591-600, Dec. 1968.

[9] H.D. Knetsch, "Mode converters and filters with rectangular and circular waveguide elements" (in German), NTZ, pp. 57-62, Feb. 1970.

[10] R.H. MacPhie and K.-L. Wu, "Scattering at the junction of a rectangular waveguide and a larger circular waveguide", IEEE Trans. Microwave Theory Tech., vol. 43, pp. 2041-2045, Sep. 1995.

[11] K. Madsen, H. Schaer-Jacobsen and J. Voldby, "Automated minimax design of networks", IEEE Trans. Circuits Systems, vol. CAS-22, pp. 791-796, Oct. 1975.

[12] U. Rosenberg, J. Bornemann and S. Amari, "Design of dual-band waveguide transformers", in 2005 IEEE MTT-S Int. Microwave Symp. Dig., WEPI-3, 4p., Long Beach, USA, June 2005. 